\title{
Angioplastia coronaria en arteria circunfleja con nacimiento anómalo en paciente con implante previo de válvula aórtica percutánea
}

\author{
Anomalous circumflex coronary artery angioplasty in a patient with \\ previous transcutaneous valve replacement.
}

Gustavo J. Moles', Oscar Birollo', Julián Dalurzo', Víctor P. Moles

\section{RESUMEN}

El reemplazo valvular percutáneo (RVP) es un procedimiento que ha generado un gran avance en el tratamiento de los pacientes con estenosis aórtica severa sintomática. Si bien comenzó demostrando su utilidad en los pacientes que no eran candidatos a una cirugía de reemplazo valvular aórtico, este procedimiento ha avanzado hacia pacientes de menor riesgo quirúrgico ${ }^{1-4}$

La prevalencia de enfermedad coronaria en pacientes con estenosis aórtica severa sintomática que eran candidatos a RVP es del $40 \%$ al $75 \%{ }^{5}$.

Debido a la naturaleza progresiva de esta enfermedad, es de esperar que muchos de estos pacientes vayan a requerir una intervención coronaria en el futuro.

Si bien se ha documentado la factibilidad de la cateterización coronaria luego del RVP, se ha descripto dificultades, en particular con válvulas de tipo autoexpandible ${ }^{6}$.

Palabras clave: reemplazo valvular aórtico percutáneo, nacimiento anómalo coronario, angioplastia coronaria, angioplastia post reemplazo valvular percutáneo.

\section{ABSTRACT}

Transcatheter aortic valve replacement (TAVR) is a procedure that has generated great advance in the treatment of patients with severe symptomatic aortic stenosis. Although it began demonstrating its usefulness in patients who were not candidates for aortic valve replacement surgery, this procedure has progressed towards patients with lower surgical risk1-4.

The prevalence of coronary disease in patients with severe symptomatic Aortic stenosis who were candidates for TAVR is $40 \%$ to $75 \% 5$.

Due to the progressive nature of this disease, it is expected that many of these patients will require coronary intervention in the future.

Although the feasibility of coronary catheterization after TAVR has been documented difficulties have been described, particularly with self-expanding valves6.

Keywords: transcatheter aortic valve replacement, coronary angioplasty after TAVR, anomalous coronary origin.

Revista Argentina de Cardioangiología Intervencionista 2018;9(4):35-36. DOI: 10.30567/RACI/201804/0035-0036

\section{CASO CLÍNICO}

Paciente de 86 años, que ingresó a unidad coronaria por cuadro de angina inestable. Como antecedentes patológicos la paciente presentaba hipertensión arterial, dislipemia, tabaquismo previo, EPOC y alergia a la penicilina.

En junio de 2016, por presentar estenosis aórtica severa y cardiopatía isquémica, se le había realizado angioplastia coronaria en arterias descendente anterior y circunfleja y luego, a los 15 días, un reemplazo de válvula aórtica de forma percutánea con válvula autoexpandible (CoreValve, MEDTRONIC)

Se solicitó cinecoronariografía diagnóstica que evidenció arteria descendente anterior con lesión leve en segmento proximal y stent permeable sin reestenosis en tercio medio, sin visualización de arteria circunfleja (Figura 1). Al cateterizar el ostium de la coronaria derecha se evidenció que dicha arteria no presentaba lesiones significativas. Además se observó nacimiento anómalo de arteria circunfleja desde segmento proximal de coronaria derecha. En esta arteria se pudo observar una lesión en segmento proximal que producía una estenosis de la luz del 90\%. Posterior a dicha lesión se encon-

1. 1. Servicio de Cardiología Intervencionista. Sanatorio San Gerónimo, Santa Fe, Argentina

$\triangle$ Correspondencia: Dr. Oscar Birollo. Av. Freyre 3068. (3000) Santa Fe, Santa Fe Argentina.oebirollo@gmail.com

Los autores no declaran conflictos de intereses

Recibido: 12/09/2018|Aceptado:03/12/2018 traba stent coronario previo permeable y sin signos de reestenosis (Figura 2).

Se continuó con angioplastia coronaria vía radial derecha a dicha lesión (cateterización selectiva de ostium de coronaria derecha con catéter guía IM $6 \mathrm{Fr}$, predilatación con balón 2,0 × $20 \mathrm{~mm}$ (MAVERICK, Boston Scientific) y luego se implanta stent convencional de $2,75 \times 12 \mathrm{~mm}$ (REBEL,

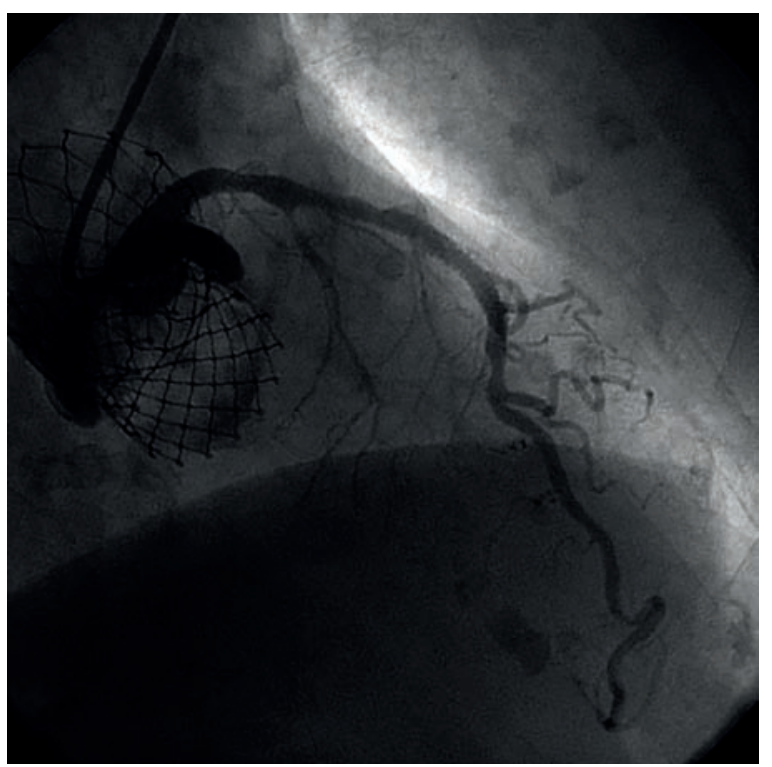

Figura 1. Visualización de arteria descendente anterior en vista oblicua izquierda caudal visualizada a través de struts de válvula autoexpandible. 


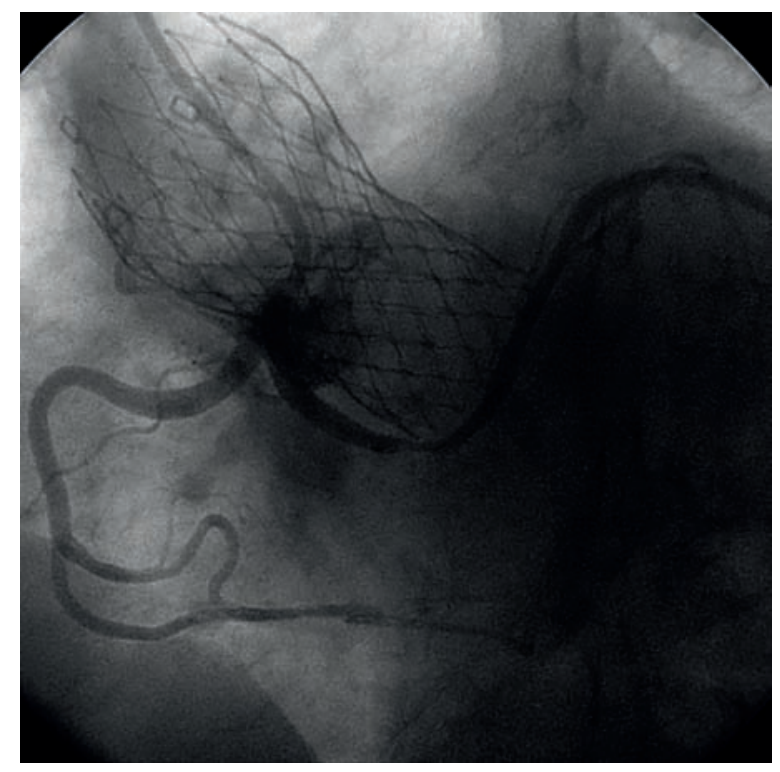

Figura 2. Visualización de arterias coronaria derecha y circunfleja mediante cateterización de ostium coronario derecho. Puede observarse estenosis del $90 \%$ a nivel proximal (flecha).

Boston Scientific). Dicho procedimiento fue exitoso y no presentó complicaciones (Figura 3).

Posteriormente la paciente cursó internación en unidad coronaria para monitoreo y fue dada de alta a las 24 hs continuando su seguimiento de forma ambulatoria.

\section{DISCUSIÓN}

La incidencia de enfermedad coronaria en pacientes a los que se les realiza un RVP es alta, aun en los de moderado riesgo. La cercanía y el aposicionamiento de los struts de la válvula generan dificultad para la cateterización selectiva del ostium coronario, especialmente con las válvulas de tipo autoexpandible.

Debemos estar preparados para superar las nuevas dificultades técnicas que se nos presentan en estos casos, aun en centros donde no se practican este tipo de procedimientos.

\section{BIBLIOGRAFÍA}

1. Leon MB, Smith CR, Mack M, et al., PARTNER Trial Investigators. Transcatheter aortic-valve implantation for aortic stenosis in patients who cannot undergo surgery. N Engl J Med 2010;363:1597-607.

2. Smith CR, Leon MB, Mack MJ, et al., PARTNER Trial Investigators. Transcatheterversus surgical aortic-valvereplacementin high-riskpatients. NEngl J Med 2011;364:2187-98.

3. Leon MB, Smith CR, MackMJ, etal., PARTNER2 Investigators. Transcatheter or surgical aorticvalve replacement in intermediate-risk patients. N Engl J Med 2016;374:1609-20.

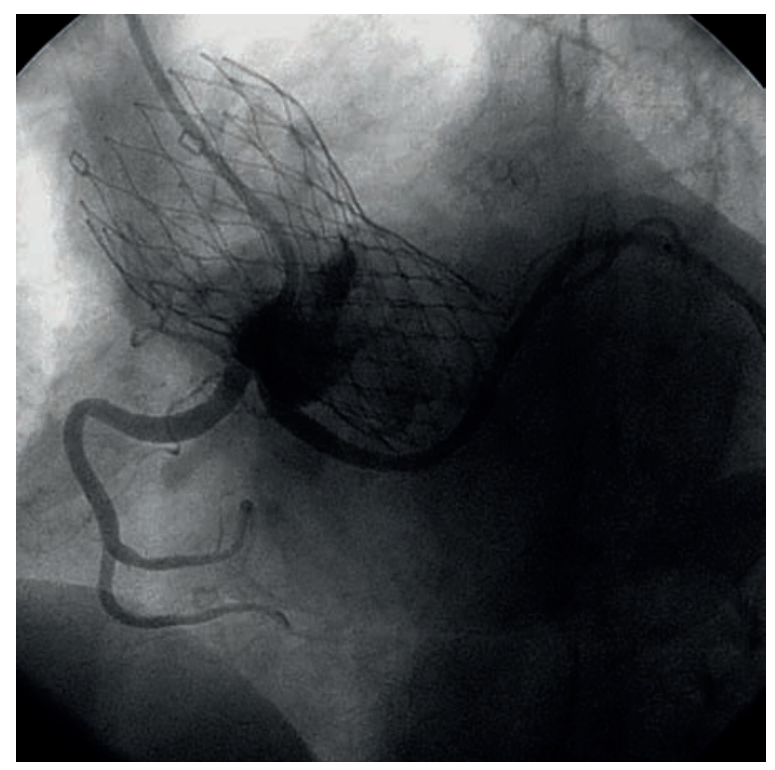

Figura 3. Resultado final post angioplastia de arteria circunfleja. Se observa resolución de la estenosis sin evidencia de signos de disección arterial (flecha)

Como es de esperarse, han sido descriptos mayores tiempos de fluoroscopia, dosis de radiación y mayor uso de contraste para estos procedimientos.

En situaciones como el síndrome coronario agudo con elevación del ST, la urgencia del cuadro clínico amerita una rápida resolución de estas dificultades, por lo que el conocimiento de la anatomía de los distintos tipos de válvulas, elementos y técnicas adecuadas es crucial a la hora de resolver el problema.

En casos programados, la tomografía multicorte puede ser útil para conocer la anatomía, identificar las dificultades y planear el acceso más adecuado a las coronarias.

Con respecto a las válvulas, es crucial la profundidad de implantación especialmente en pacientes donde el ostium nace a menos de $10 \mathrm{~mm}$ del anillo.

Esperamos que con mayor experiencia y desarrollos tecnológicos estas prótesis puedan ser modificadas para facilitar la cateterización selectiva de las arterias coronarias y de esta manera descomplejizar estos procedimientos en pacientes de este tipo.

4. Reardon MJ, Van Mieghem NM, Popma JJ, et al., SURTAVI Investigators. Surgical or transcatheter aortic-valve replacement in intermediate-risk patients. N Engl J Med 2017; 376:1321-31.

5. Goel SS, Ige M, Tuzcu EM, et al. Severe aortic stenosis and coronary artery disease-implications for management in the transcatheter aortic valve replacementera: a comprehensive review. J Am Coll Cardiol 2013;62:1-10.

6. Boukantar M, Gallet R, Mouillet G, et al. Coronary procedures after TAVI with the self-expanding aortic bioprosthesis Medtronic CoreValve, not an easy matter. J Interv Cardiol 2017;30:56-62. 